\title{
Asymptotic Dichotomy in a Class of Fourth-Order Nonlinear Delay Differential Equations with Damping
}

\author{
Chengmin Hou' ${ }^{1}$ and Sui Sun Cheng ${ }^{2}$ \\ ${ }^{1}$ Department of Mathematics, Yanbian University, Yanji 133002, China \\ ${ }^{2}$ Department of Mathematics, Tsing Hua University, Hsinchu 30043, Taiwan \\ Correspondence should be addressed to Sui Sun Cheng, sscheng@math.nthu.edu.tw \\ Received 5 December 2008; Revised 15 February 2009; Accepted 26 February 2009 \\ Recommended by Agacik Zafer
}

All solutions of a fourth-order nonlinear delay differential equation are shown to converge to zero or to oscillate. Novel Riccati type techniques involving third-order linear differential equations are employed. Implications in the deflection of elastic horizontal beams are also indicated.

Copyright (C) 2009 C. Hou and S. S. Cheng. This is an open access article distributed under the Creative Commons Attribution License, which permits unrestricted use, distribution, and reproduction in any medium, provided the original work is properly cited.

\section{Introduction}

It has been observed that the solutions of quite a few higher order functional differential equations oscillate or converge to zero (see, e.g., the recent paper [1]). Such a dichotomy may sometimes yield useful information in real problems (see the remark at the end of this note). In this paper, we report that the same phenomena occurs for fourth-order nonlinear delay differential equations of the form

$$
y^{(4)}(t)+p(t) y^{\prime}(t)+q(t) f(y(g(t)))=0, \quad t \in I:=[a, \infty),
$$

where

(i) $a$ is a nonnegative constant, $p$ is a nonnegative function in $C(I, R)$ such that it does not vanish identically on any $[T, \infty) \subseteq I$;

(ii) $q$ is a positive function in $C(I, R)$;

(iii) $g \in C^{1}(I, R)$ and $0<g(t)<\mathrm{t}, g^{\prime}(t) \geq 0$ for $t \in I$ and $\lim _{t \rightarrow+\infty} g(t)=+\infty$;

(iv) $f \in C(R, R)$ and $f(u) / u \geq \kappa>0$ for $u \neq 0$. 
As is well known, fourth-order differential equations can be used to model the deflection of a beam (in particular, the function $y(t)$ in (1.1) may be interpreted as the deflection from the equilibrium position of a horizontal beam at the spatial coordinate $t$ ). Therefore, a short note reporting our observation may be of interest in the qualitative theory of fourth-order differential equations. Indeed, in this note, we will distinct ourselves by emphasizing the role played by the function $p$ in (1.1), since the term $p(t) y^{\prime}(t)$ acts as a control of the slope of the beam under consideration at the coordinate $t$.

We will restrict our attention to those solutions of (1.1) which exist on $I$ and satisfy the condition $\sup \{|y(t)|: T \leq t<\infty\}>0$ for any $T \in I$. Such a solution is called oscillatory if it has arbitrarily large zeros, and nonoscillatory otherwise.

Lemma 1.1. Assume that $x \in C^{n}(I, R)$ such that $x(t)>0$ for $t \geq a$ and $x^{(n)}(t)$ is nonpositive for $t \geq a$ and does not vanish identically on any $[T, \infty) \subseteq$ I. If $n$ is even (or odd), then there exists $l \in\{1,3, \ldots, n-1\}$ (resp., $l \in\{0,2, \ldots, n-1\}$ ) such that for all sufficiently large $t, x(t) x^{(j)}(t)>0$ for $j=0,1, \ldots, l$, and $(-1)^{n+j-1} x(t) x^{(j)}(t)>0$ for $j=l+1, l+2, \ldots, n-1$. Furthermore,

$$
\left|x^{\prime}(g(t))\right| \geq \frac{g^{l-1}(t)(t-g(t))^{n-l-1}}{2^{l-1}(l-1) !(n-1-l) !}\left|x^{(n-1)}(t)\right|
$$

for all sufficiently large $t$, where $g \in C^{1}(I, R)$ satisfies $0<g(t)<t, \quad g^{\prime}(t) \geq 0$ and $\lim _{t \rightarrow \infty} g(t)=$ $+\infty$.

Proof. The existence of $l$ in the above result is due to Kiguradze and is well known (see, e.g., [2]). Next, we will prove the inequality (1.2) for even $n$ (the odd case being similar) as follows (cf. [3]). First we may assume that there is a $T>0$ such that $x(t)>0, x^{\prime}(t)>0, \ldots, x^{(l)}(t)>$ $0,(-1)^{n+l} x^{(l+1)}(t)>0, \ldots,(-1)^{2 n-2} x^{(n-1)}(t)>0$ for $t \geq T$. By Taylor's formula, we have

$$
x^{\prime}(s)=x^{\prime}(T)+x^{\prime \prime}(T)(s-T)+\cdots+\frac{x^{(l)}\left(s^{*}\right)}{(l-1) !}(s-T)^{l-1} \geq \frac{x^{(l)}\left(s^{*}\right)}{(l-1) !}(s-T)^{l-1},
$$

where $T \leq s^{*} \leq s$. Furthermore,

$$
x^{\prime}(s) \geq \frac{x^{(l)}(s)}{(l-1) !}(s-T)^{l-1}, \quad s \geq T,
$$

since $x^{(l+1)}(t)<0$ for $t \in[T,+\infty)$. Next, we pick $T_{1} \geq T$ such that $1-T / g(t)>1 / 2$ for $t \geq T_{1}$. Then $g(t)>2 T$ and

$$
x^{\prime}(g(t)) \geq \frac{x^{(l)}(g(t))}{(l-1) !} g^{l-1}(t)\left(1-\frac{T}{g(t)}\right)^{l-1} \geq \frac{g^{l-1}(t)}{2^{l-1}(l-1) !} x^{(l)}(g(t)), \quad t \geq T_{1} .
$$

By Taylor's formula again, we get

$$
x^{(l)}(g(t))=x^{(l)}(t)+(-1) x^{(l+1)}(t)(t-g(t))+\cdots+(-1)^{n-l-1} \frac{x^{(n-1)}\left(t^{*}\right)}{(n-l-1) !}(t-g(t))^{n-l-1},
$$


here $g(t) \leq t^{*} \leq t$. Hence

$$
x^{(l)}(g(t)) \geq \frac{x^{(n-1)}\left(t^{*}\right)}{(n-l-1) !}(t-g(t))^{n-l-1} \geq \frac{x^{(n-1)}(t)}{(n-l-1) !}(t-g(t))^{n-l-1} .
$$

Combining (1.7) and (1.5), we see that

$$
x^{\prime}(g(t)) \geq \frac{g^{l-1}(t)}{2^{l-1}(l-1) !(n-l-1) !} x^{(n-1)}(t)(t-g(t))^{n-l-1}
$$

for all large $t$.

Lemma 1.2. Suppose the linear third-order differential equation

$$
z^{\prime \prime \prime}+p(t) z=0, \quad t \in I
$$

has an eventually positive increasing solution on I and $y$ is a nonoscillatory solution of (1.1) on I. Then there exists $t_{0} \in[a, \infty)$ such that $y(t) y^{\prime}(t)>0$ or $y(t) y^{\prime}(t)<0$ for $t \geq t_{0}$.

Proof. Without loss of generality, we may assume that $y(t)>0$ and $y(g(t))>0$ for $t \geq a$. Then $x(t)=-y^{\prime}(t)$ is a solution of the third-order delay differential equation

$$
x^{\prime \prime \prime}+p(t) x=q(t) f(y(g(t))), \quad t \geq a .
$$

We claim that, all solutions of (1.10) are nonoscillatory (so that $y^{\prime}(t)$ is eventually positive or eventually negative). To see this, let $z$ be the solution of (1.9) such that $z(t)>0$ and $z^{\prime}(t)>0$ for $t \geq a$. Then from Lemma 1.1, we have $z^{\prime \prime}(t)>0$ for $t$ greater than or equal to a positive number $t_{1}$.

Suppose to the contrary that $x$ is an oscillatory solution of (1.10). We assert that $s=$ $x^{\prime} z-x z^{\prime}$ oscillates. Indeed, since $x$ is oscillatory, for any $T>a$, there are $b$ and $c$ such that $T \leq b<c, x(b)=x(c)=0, x^{\prime}(b) \geq 0$ and $x^{\prime}(c) \leq 0$. Hence $s(b)=x^{\prime}(b) z(b) \geq 0$ and $s(c)=x^{\prime}(c) z(c) \leq 0$. If $s(b)=0$ or $s(c)=0$, then either $b$ or $c$ is a zero of $s$ in $[T, \infty)$. Otherwise, $s(b)>0$ and $s(c)<0$ so that there is $d \in(b, c)$ such that $s(d)=0$. These show that $s$ has arbitrarily large zeros.

Now that $s$ is oscillatory, either $s(t) \leq 0$ for all $t \in\left[t_{1}, \infty\right)$ or else there are strictly increasing and divergent sequences $\left\{b_{n}\right\}$ and $\left\{c_{n}\right\}$ such that $s(t)>0$ for $t \in\left(b_{n}, c_{n}\right) \subset$ $\left(t_{1}, \infty\right), s\left(b_{n}\right)=s\left(c_{n}\right)=0, s^{\prime}\left(b_{n}\right) \geq 0$ and $s^{\prime}\left(c_{n}\right) \leq 0$.

Suppose the former case holds. Then there are $(b, c) \subset\left(t_{1}, \infty\right)$ such that $s(t) \leq 0$ for $t \in(\mathrm{b}, c)$ and $s^{\prime}(b) \geq 0$ and $s^{\prime}(c) \leq 0$. From (1.9) and (1.10), we have

$$
z q(t) f(y(g(t)))=x^{\prime \prime \prime} z-z^{\prime \prime \prime} x=s^{\prime \prime}-\frac{z^{\prime}}{z} s^{\prime}+\frac{z^{\prime \prime}}{z} s,
$$

or,

$$
\left(\frac{s^{\prime}}{z}\right)^{\prime}=q(t) f(y(g(t)))-\frac{z^{\prime \prime} s}{z^{2}}
$$


Hence we have

$$
0 \geq \int_{b}^{c}\left(\frac{s^{\prime}(t)}{z(t)}\right)^{\prime} d t=\int_{b}^{c}\left\{q(t) f(y(g(t)))-\frac{z^{\prime \prime}(t) s(t)}{z^{2}(t)}\right\} d t>0,
$$

which is a contradiction.

Suppose the latter case holds. From (1.9) and (1.10), we have

$$
x^{\prime \prime \prime} z^{\prime}-z^{\prime \prime \prime} x^{\prime}+p(t)\left(x z^{\prime}-x^{\prime} z\right)=z^{\prime} q(t) f(y(g(t))),
$$

from which by

$$
x^{\prime \prime \prime} z^{\prime}-z^{\prime \prime \prime} x^{\prime}=\left(\frac{z^{\prime}}{z} s^{\prime}-\frac{z^{\prime \prime}}{z} s\right)^{\prime}
$$

we obtain

$$
\left(\frac{z^{\prime}}{z} s^{\prime}-\frac{z^{\prime \prime}}{z} s\right)^{\prime}-p(t) s=z^{\prime} q(t) f(y(g(t)))
$$

Since $z^{\prime}(t)>0, s^{\prime}\left(c_{n}\right) \leq 0, s^{\prime}\left(b_{n}\right) \geq 0, s\left(c_{n}\right)=s\left(b_{n}\right)=0, s(t)>0, t \in\left(b_{n}, c_{n}\right)$, we see that

$$
\begin{aligned}
0 & <\int_{b_{n}}^{c_{n}} z^{\prime}(t) q(t) f(y(g(t))) d t \\
& =\int_{b_{n}}^{c_{n}}\left\{\left(\frac{z^{\prime}(t)}{z(t)} s^{\prime}(t)-\frac{z^{\prime \prime}(t)}{z(t)} s(t)\right)^{\prime}-p(t) s(t)\right\} d t \\
& =\frac{z^{\prime}\left(c_{n}\right)}{z\left(c_{n}\right)} s^{\prime}\left(c_{n}\right)-\frac{z^{\prime}\left(b_{n}\right)}{z\left(b_{n}\right)} s^{\prime}\left(b_{n}\right)-\int_{b_{n}}^{c_{n}} p(t) s(t) d t \\
& \leq 0
\end{aligned}
$$

which is a contradiction.

\section{Asymptotic Dichotomy}

We are now ready to state and prove our main result.

Theorem 2.1. Suppose that

(i) equation (1.9) has an eventually positive increasing solution $z$ on $[a, \infty)$,

(ii) there is a differentiable function $\rho:[a, \infty) \rightarrow(0, \infty)$ such that

$$
\limsup _{t \rightarrow \infty} \int_{T}^{t}\left\{\kappa \rho(s) q(s)-\frac{(l-1) !(3-l) !\left(\rho^{\prime}(s)\right)^{2}}{2^{3-l} g^{l-1}(s)(s-g(s))^{3-l} g^{\prime}(s) \rho(s)}\right\} d s=+\infty, \quad l=1 \text { and } 3,
$$

for every $T \geq a$, 
(iii)

$$
\limsup _{t \rightarrow \infty} \int_{T}^{t} \int_{T}^{s}\{\kappa q(u)-p(u)\} d u d s=+\infty
$$

Then every solution $y$ of (1.1) either oscillates or converges to 0.

Proof. Let $y$ be a solution of (1.1) which does not converge to 0 . Suppose to the contrary that $y$ is a nonoscillatory solution of (1.1). Without loss of generality, we may assume that $y(t)>0$ and $y(g(t))>0$ for $t \geq a$. Furthermore, by Lemma 1.2, there is $t_{0} \geq a$ such that $y^{\prime}(t)>0$ or $y^{\prime}(t)<0$ for $t \geq t_{0}$.

Suppose $y^{\prime}(t)>0$ for $t \geq t_{0}$. Then by (1.1),

$$
y^{(4)}(t)=-p(t) y^{\prime}(t)-q(t) f(y(g(t)))<0, \quad t \geq t_{0} .
$$

By Lemma 1.1, we may suppose further that $y^{\prime \prime \prime}(t)>0$ and (1.2) holds for $t \geq t_{0}$ as well. Let

$$
\omega(t):=\frac{\rho(t) y^{\prime \prime \prime}(t)}{y(g(t))}>0, \quad t \geq t_{0} .
$$

Then by (1.1) and (1.2), we see that

$$
\begin{aligned}
\omega^{\prime}(t) \leq & -\kappa \rho(t) q(t)+\frac{\rho^{\prime}(t)}{\rho(t)} \omega(t)-\frac{\rho(t) y^{\prime \prime \prime}(t) y^{\prime}(g(t)) g^{\prime}(t)}{y^{2}(g(t))} \\
\leq & -\kappa \rho(t) q(t)+\frac{\rho^{\prime}(t)}{\rho(t)} \omega(t)-\frac{g^{l-1}(t)(t-g(t))^{3-l} g^{\prime}(t)}{2^{l-1}(l-1) !(3-l) ! \rho(t)} \omega^{2}(t) \\
= & -\kappa \rho(t) q(t)-\frac{g^{l-1}(t)(t-g(t))^{3-l} g^{\prime}(t)}{2^{l-1}(l-1) !(3-l) ! \rho(t)}\left(\omega-\frac{2^{l-1}(l-1) !(3-l) ! \rho^{\prime}(t)}{2 g^{l-1}(t)(t-g(t))^{3-l} g^{\prime}(t)}\right)^{2} \\
& +\frac{(l-1) !(3-l) !\left(\rho^{\prime}(t)\right)^{2}}{2^{3-l} g^{l-1}(t)(t-g(t))^{3-l} g^{\prime}(t) \rho(t)} \\
\leq & -\left\{\kappa \rho(t) q(t)-\frac{(l-1) !(3-l) !\left(\rho^{\prime}(t)\right)^{2}}{2^{3-l} g^{l-1}(t)(t-g(t))^{3-l} g^{\prime}(t) \rho(t)}\right\}, \quad t \geq \alpha .
\end{aligned}
$$

Hence

$$
\begin{aligned}
& \int_{t_{0}}^{t}\left\{\kappa \rho(t) q(t)-\frac{(l-1) !(3-l) !\left(\rho^{\prime}(t)\right)^{2}}{2^{3-l} g^{l-1}(t)(t-g(t))^{3-l} g^{\prime}(t) \rho(t)}\right\} d s \\
& \quad \leq-\int_{t_{0}}^{t} \omega^{\prime}(s) d s=\omega\left(t_{0}\right)-\omega(t) \leq \omega\left(t_{0}\right)
\end{aligned}
$$

for all $t \geq t_{0}$, which is contrary to our assumption (2.1). 
Suppose $y^{\prime}(t)<0$ for $t \geq t_{0}$. Since $y$ is eventually positive, eventually decreasing, and does not converge to 0 , we easily see that (i) $y^{\prime \prime}$ cannot be eventually nonpositive, (ii) $\lim _{t \rightarrow \infty} y(t)=\lambda>0$, (iii) $\lim _{t \rightarrow \infty} y^{\prime}(t)=0$, and (iv) there is $\alpha \geq a$ such that $-y^{\prime}(t)<\lambda<y(t)$ for $t \geq \alpha$.

In view of (i) above, either $y^{\prime \prime}$ is oscillatory or $y^{\prime \prime}$ is eventually nonnegative. If $y^{\prime \prime}$ is oscillatory, then $y^{\prime \prime \prime}$ is also oscillatory. Hence there exists some number $\beta \geq \alpha$ such that $y^{\prime \prime \prime}(\beta)=0$ and $y(g(t)) \geq \lambda$ for $t \geq \beta$. From (1.1) and (2.2), we have

$$
y^{\prime \prime \prime}(t)=-\int_{\beta}^{t} y(g(s)) q(s) \frac{f(y(g(s)))}{y(g(s))} d s-\int_{\beta}^{t} p(s) y^{\prime}(s) d s \leq-\lambda \int_{\beta}^{t}[\kappa q(s)-p(s)] d s .
$$

It follows that

$$
y^{\prime \prime}(t) \leq y^{\prime \prime}(\beta)-\lambda \int_{\beta}^{t} \int_{\beta}^{s}[\kappa q(u)-p(u)] d u d s
$$

Since the right-hand side tends to $-\infty$ as $t$ tends to $+\infty, y^{\prime \prime}$ is eventually negative which is contrary to our assumption on $y^{\prime \prime}$.

As a consequence, we must have $\left(y(t)>0, y^{\prime}(t)<0\right.$ and $) y^{\prime \prime}(t) \geq 0$, say for $t \geq \alpha \geq a$. There are three cases to consider:

Case 1. If $y^{\prime \prime \prime}(t) \geq 0$ and does not vanish identically for $t \geq \alpha$, then $y^{\prime}(t)>0$ for all large $t$, a contradiction. If $y^{\prime \prime \prime}(t) \equiv 0$ for $t \geq \alpha$, then $y^{\prime}$ is a linear function. Since $\lim _{t \rightarrow \infty} y^{\prime}(t)=0$, we get $y^{\prime}(t)=0$ for $t \geq \alpha$, which is contrary to our assumption that $y^{\prime}(t)<0$ for $t \geq \alpha$.

Case 2. If $y^{\prime \prime \prime}(t) \leq 0$ for $t \geq \alpha$, then from (1.1), we get

$$
\begin{aligned}
y^{\prime \prime \prime}(\alpha)-y^{\prime \prime \prime}(t) & =\int_{\alpha}^{t} q(s) f(y(g(s))) d s+\int_{\alpha}^{t} p(s) y^{\prime}(s) d s \geq \lambda \int_{\alpha}^{t}(\kappa q(s)-p(s)) d s, \\
0 & \geq-y^{\prime \prime}(t) \geq-y^{\prime \prime}(\alpha)+\lambda \int_{\alpha}^{t} \int_{\alpha}^{s}(\kappa q(\tau)-p(\tau)) d \tau d s,
\end{aligned}
$$

which is contrary to (2.2).

Case 3. If $y^{\prime \prime \prime}$ oscillates, then (2.8) holds so that $y^{\prime \prime}$ is eventually negative, which is contrary to our assumption on $y^{\prime \prime}$. This completes the proof.

\section{Remarks and Example}

We remark that the condition (2.2) in the above result can, in case $p$ is differentiable, be replaced by the alternate condition

$$
\limsup _{t \rightarrow \infty} \int_{T}^{t}\left\{\kappa q(u)-p^{\prime}(u)\right\} d u=+\infty
$$


for every $T \geq 0$. Indeed, we only need to note that

$$
\begin{aligned}
& \int_{\gamma}^{t} q(s) f(y(g(s))) d s+\int_{\gamma}^{t} p(s) y^{\prime}(s) d s \\
& \quad= \int_{\gamma}^{t} y(g(s))\left[q(s) \frac{f(y(g(s)))}{y(g(s))}\right] d s+\left.p(s) y(s)\right|_{\gamma} ^{t}-\int_{\gamma}^{t} y(s) p^{\prime}(s) d s \\
& \quad \geq \lambda \int_{\gamma}^{t}\left[\kappa q(s)-p^{\prime}(s)\right] d s-p(\gamma) y(\gamma)
\end{aligned}
$$

where $\gamma=\alpha$ or $\beta$, and then we may follow the rest of the above arguments.

Example 3.1. Consider the fourth-order delay differential equation

$$
y^{(4)}+\frac{1}{t^{3}(\ln t-1)} y^{\prime}+\frac{1}{t^{2}}\left(1-\frac{1}{t(\ln t-1)}\right) y\left(t-\frac{3 \pi}{2}\right)=0, \quad t>\frac{3 \pi}{2} .
$$

Here, $p(t)=1 / t^{3}(\ln t-1), q(t)=\left(1 / t^{2}\right)(1-1 / t(\ln t-1))$, and $f(u)=u$ with $\kappa=1$. It is clear that (2.2) is satisfied. The equation $z^{\prime \prime \prime}+\left(1 / t^{3}(\ln t-1)\right) z=0$ has an eventually positive and eventually increasing solution $z=t(\ln t-1)$. By choosing $\rho(t)=t$ and any large $T$, we have

$$
\limsup _{t \rightarrow \infty} \int_{T}^{t}\left[\left(\frac{1}{s^{2}}-\frac{1}{s^{3}(\ln s-1)}\right) s-\frac{(l-1) !(3-l) !}{2^{3-l} s(s-3 \pi / 2)^{l-1}(3 \pi / 2)^{3-l}}\right] d s=+\infty, \quad l=1 \text { and } 3 .
$$

Consequently, condition (2.1) is satisfied. Hence by Theorem 2.1, any solution of (3.3) is oscillatory or satisfies $\lim _{t \rightarrow \infty} y(t)=0$.

As our final remark, suppose we have a (sufficiently) long elastic horizontal beam modeled by (1.1). Our Theorem says that (under appropriate conditions) if the right end of the beam is fixed at some nonzero vertical position, then there must be places where there are positive as well as negative displacements of the beam from its equilibrium position. This shows that the dichotomy in our Theorem has implications in the elastic beam deflection problem.

\section{References}

[1] A. Tiryaki and M. F. Aktaş, "Oscillation criteria of a certain class of third order nonlinear delay differential equations with damping," Journal of Mathematical Analysis and Applications, vol. 325, no. 1, pp. 54-68, 2007.

[2] G. S. Ladde, V. Lakshmikantham, and B. G. Zhang, Oscillation Theory of Differential Equations with Deviating Arguments, vol. 110 of Monographs and Textbooks in Pure and Applied Mathematics, Marcel Dekker, New York, NY, USA, 1987.

[3] Z. G. Ouyang and Y. K. Li, “Monotone solutions of even-order delay differential equations," Journal of Mathematical Research and Exposition, vol. 24, no. 2, pp. 321-327, 2004. 


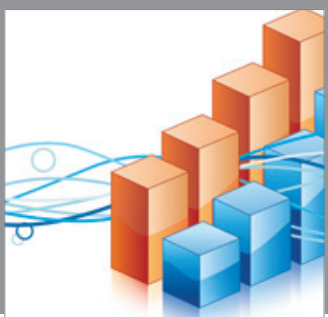

Advances in

Operations Research

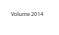

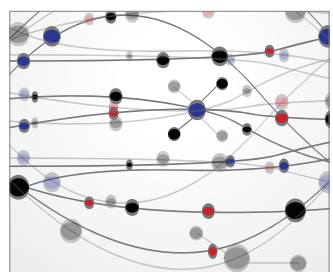

\section{The Scientific} World Journal
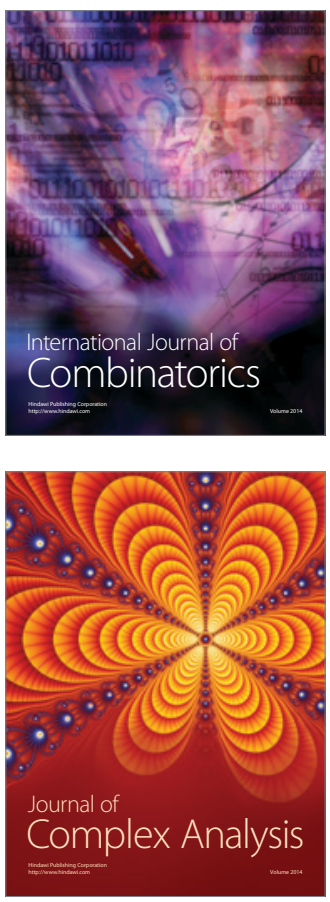

International Journal of

Mathematics and

Mathematical

Sciences
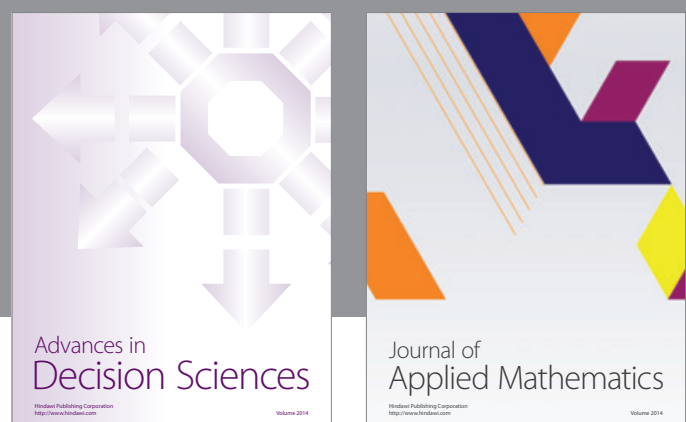

Journal of

Applied Mathematics
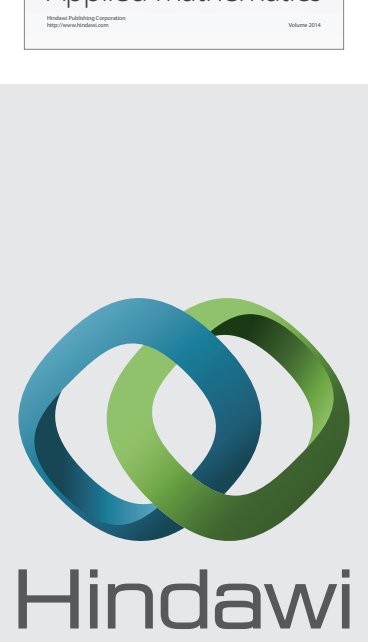

Submit your manuscripts at http://www.hindawi.com
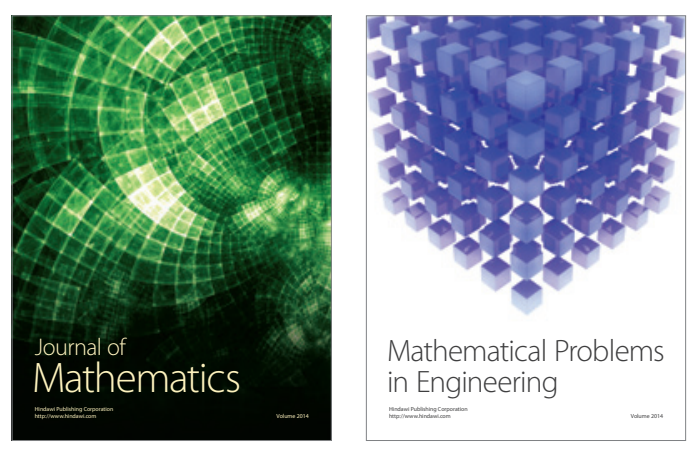

Mathematical Problems in Engineering
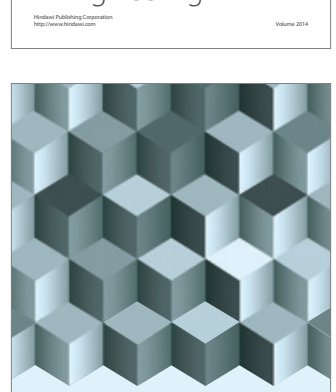

Journal of

Function Spaces
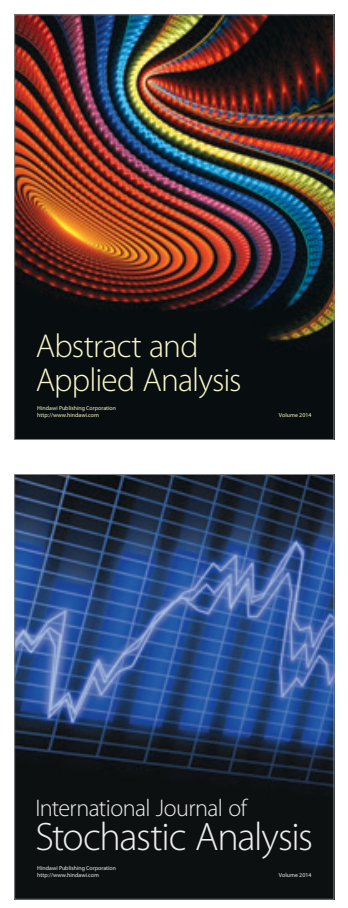

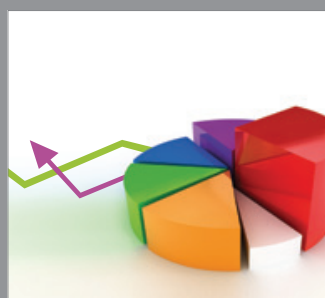

ournal of

Probability and Statistics

Promensencen
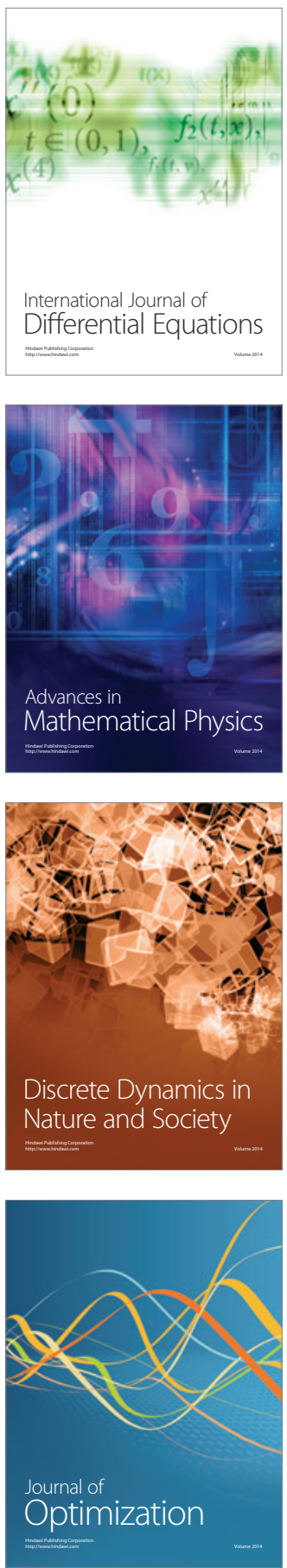